\title{
Land Cover Feature Extraction and Analysis using Biogeography based Optimization (BBO) Algorithm
}

\author{
Prabhjot Kaur \\ M.tech Scholar \\ GNDEC LUDHIANA
}

\author{
Kamaljit Kaur \\ Dhillon \\ Assistant Professor \\ GNDEC LUDHIANA
}

\author{
Rajdeep Singh \\ Chauhan \\ Assistant Professor \\ LLRIET, MOGA
}

\author{
Savneet Kaur \\ Assistant Professor \\ LLRIET, MOGA
}

\begin{abstract}
The entropy vector is used to identify the homogeneity between the regions but in this proposed approach we are using a wavelet inspired segmented approach for the region match so that more clearly differentiate the homogenous and heterogeneous regions over the image. In this existing work, the $\mathrm{K}$-means clustering is used as the initial classification and then the BBO is implemented. An initial segmented analysis is implemented to identify the number of classes more accurately and then the classification is performed. The main objective of the work is to perform the region classification for the land cover images development and implement wavelet inspired BBO approach to perform the classification process. The initial segmentation based similarity measure is performed to identify the number of classes using Clustering method and then the clustering process is done using Cmeans. The major objective of the work is to define more efficient classification under different regions.
\end{abstract}

Keywords: Image Segmentation, BBO, K-Means Clustering

\section{INTRODUCTION}

The image segmentation divides the given image into its constituents parts depending upon the property or feature used for decomposing the same. Typically, image segmentation is used for upbringing the region of interest from the given image.

The most commonly used feature for image segmentation is the pixel color. The color format normally used in image segmentation is either RGB (24-bit color) or gray color format (8-bit color format). The color based image segmentation provides a fair degree of segmentation and serves almost many purposes. Examples can be face detection based on skin color, water/mineral rich area from satellite images,

The image segmentation partitions the given image into different regions using the user defined feature. The feature is computed form the image and then applied to segment the image under study.

\subsection{Enhancement}

Medical images are often deteriorated by noise due to interference and other phenomena that affect the imaging processes. Image enhancement is the improvement of image quality to increase the perception of information in images for medical specialists.

\subsection{Sharpening}

Enhancing the sharpness by accentuate edges may contribute to raise more visible details in an image. Laplacian, Sobel, Rebert Cross are some algorithms used to extract edges and thus increase the sharpness of the image.

\section{RELATED WORKS}

In Year 2007, Carlos Rueda performed a work," Modeling Satellite Image Streams for Change Analysis". Satellite image modelling is performed hare and then the c-means clustering is adopted to segment the images. [1]

In Year 2010, Dr.P.Subashini performed a work," Quantitative performance evaluation on segmentation methods for SAR ship images". The comparison is based on the potential performance measures.

In Year 2010, Prof. Dr. P. K. Srimani performed a work," Analysis and Evaluation of Classifiers Using Multi-Temporal Images in Land Use and Land Cover Mapping". The Kappa and Overall accuracy of MLC is much better compared to MHDC and MDC.

In Year 2009, Dr.P.Subashini performed a work, "A combined preprocessing scheme for texture based Ice classification from SAR images". This paper presents a preliminary study of image processing on the ice patterns in synthetic aperture radar (SAR) imagery.

In Year 2012, Kanchan Bahirat performed a work, "Matte Based Generation of Land Cover Maps". A novel supervised technique for the generation of spatially consistent land cover maps based on class-matting is presented in this paper. This method takes advantage of both standard supervised classification technique and natural image matting. It adaptively exploits the spatial contextual information contained in the neighborhood of each pixel through the use of image matting to reduce the incongruence inherent in pixel-wise, radiometric classification of multispectral remote sensing data, providing a more spatially homogeneous landcover map besides yielding a better accuracy.

In Year 2009, Abida Najab performed a work," Principal Component Analysis Based Classification of Settlements in Satellite Images". The objective of this research is to use satellite images for the classification and identification of settlements. Satellite images are used in this research. A wide area is covered in a single satellite image and it contains enormous information therefore satellite images can be used for many useful purposes, like classification of objects and land cover classes, change detection and population estimation etc. Many small settlements are usually scattered in remote areas.

In Year 2011, Lavika Goel performed a work," Performance Governing Factors of Biogeography Based Land Cover Feature Extraction: Biogeography based Optimization (BBO) has been used for focused land cover feature extraction.

In Year 2010, Triloki Pant performed a work," Application of Fractal Parameters for Unsupervised Classification of SAR 
images: A Simulation based Study". Classification of image is done here using fractal parameters viz. fractal dimension and lacunarity.

In Year 2010, V Turkar performed a work," Polarimetric SAR Image Classification by Using Artificial Neural Network". The classification of various land cover features using fully polarimetric Synthetic Aperture Radar (SAR) data sets is an important application of radar remote sensing. The proposed classifier is based on the artificial neural network which is developed in Matlab and it makes use of backscattering values. It is a supervised classification technique which is applied on the ALOS PALASR and SIR-C data. The classification accuracy after applying different speckle filters is compared with the classification accuracy obtained without applying filter.

In Year 2012, Bruno M. Carvalho performed a work," Texture Fuzzy Segmentation using Adaptive Affinity Functions". In this paper Author present an extension of the fuzzy segmentation algorithm that achieves the segmentation of textures by employing adaptive affinity functions. The adaptive affinity functions change the size of the area (neighborhood) where they compute the texture descriptors, according to the characteristics of the texture being processed.

In Year 2010, Ruochen Liu performed a work," A Multiobjective Immune Clustering Ensemble Technique Applied to Unsupervised SAR Image Segmentation". This paper proposes a novel unsupervised approach for synthetic aperture radar (SAR) image segmentation, namely, multiobjective immune clustering ensemble technique (MICET). The new technique first divides the image into several regions, and a certain number of pixels are picked out from these regions to form the clustering dataset. Second, artificial immune system (AIS) and multiobjective optimization (MOO) are introduced to generate multiple clustering results, which are then combined together for the following ensemble process.

In Year 2011, Jiang Li performed a work," Remote Sensing Image Information Mining with HPC Cluster and DryadLINQ". This paper presents a distributed framework for information mining from multi-dimensional remotely sensed images using Windows High Performance Computing (HPC) Servers and Dryad distributed computing engine. Land cover and land use types are classified by Support Vector Machines (SVM) and stored in an object-oriented database with region quad-tree indices.

In Year 2009, H.B. Kekre performed a work," SAR Image Segmentation using co- occurrence matrix and slope magnitude". An edge detector for SAR images is proposed. The algorithm works in horizontal as well as in vertical direction in order to compute the true edges of the image objects.

In Year 2009, Xiuli Ma performed a work," Spectral Clustering Ensemble for Image Segmentation". Multi way spectral clustering technique is used here.

In Year 2007, Jiang Li performed a work," Hierarchical Land Cover Information Retrieval in Object-Oriented Remote Sensing Image Databases with Native Queries. In Year 2012, Mark Hauschild performed a work," Image Segmentation using a Genetic Algorithm and Hierarchical Local Search".

\section{PRESENT WORK 3.1 Clustering}

Clustering segments the given image based on some properties like pixel color, location or any statistical property like standard deviation and entropy etc. The main property of a cluster is that each member of a cluster is at minimum average distance from its centre of cluster and farthest from centre of mass of other cluster. This property draws a clear cut boundary between the clusters. Further, each cluster has its own centre of mass.

The popular method of clustering is using K-means clustering, where $\mathrm{K}$ is the no. of clusters in the data set. $\mathrm{K}$ means clustering is based on minimum Euclidean distance of data objects from the initialised centre of clusters and each member is assigned to the respective cluster from where it has minimum Euclidean distance. The algorithm is iterated until unless the centre of clusters is converged to a constant point.

\subsection{Algorithm}

The presented work is about to perform the region classification for the land cover images. In this work a layered approach is defined for the classification. At the initial state the segmentation will be performed to identify the number of classes over the images. As the classes identify the C Means clustering will be performed and finally as the major stage the BBO algorithm will be implemented for the featured analysis. The work is about to identify the homogenous and the heterogeneous regions more clearly over these land cover images.

The presented work is implemented in matlab version 7.5 and can be implemented the logic in any hardware oriented language for real time image processing. The basic design for the work is given as under:-

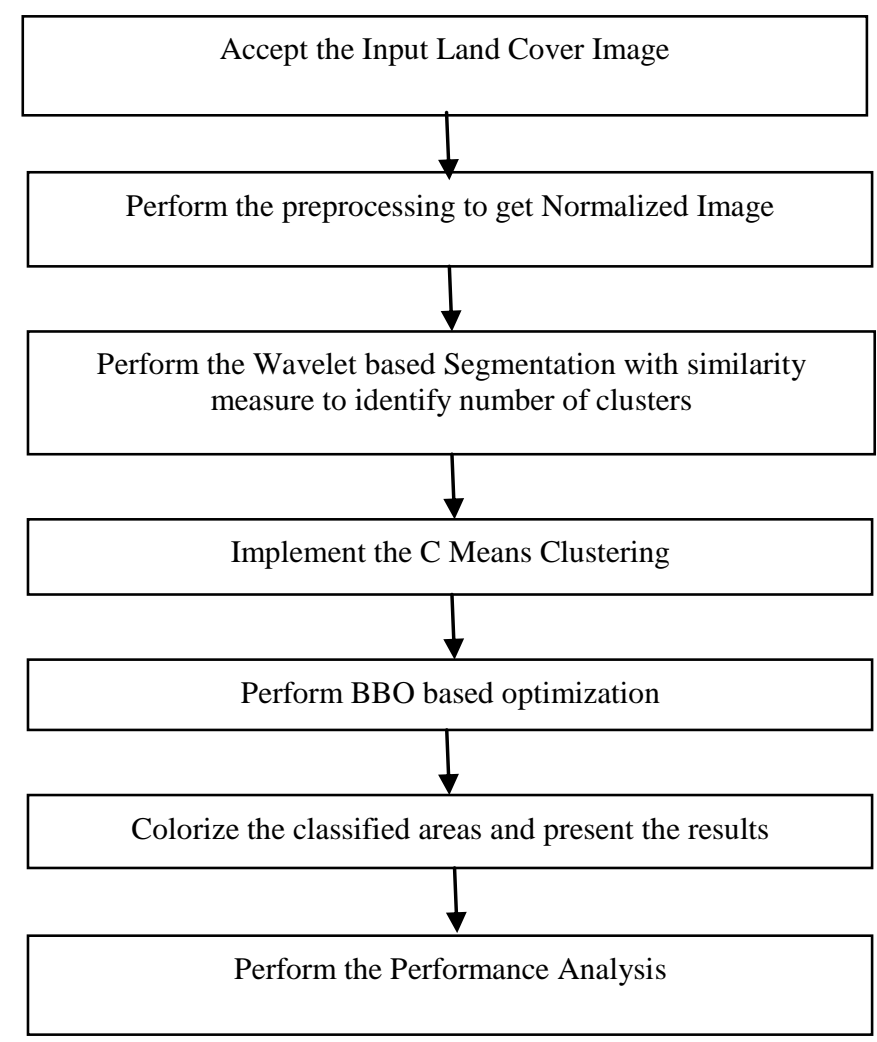

Figure 3.1 Flow Chart of BBO Algorithm 


\section{RESULTS AND CONCLUSION}

In the presented review work, some recent techniques are discussed for segmentation of satellite image for land cover analysis. Normally the images are in jpeg format i.e. 24-bit color format (RGB) and can be segmented using the same color format using color as base feature. However, a fast segmentation approach could be achieved if the jpeg image is converted to gray scale image and then apply the BBO algorithm for image segmentation and then analyze the segmented image parts using statistical analysis for feature extraction and analysis. Some snap shots of the proposed algorithm developed in matlab version 7.5 are shown below in figures.
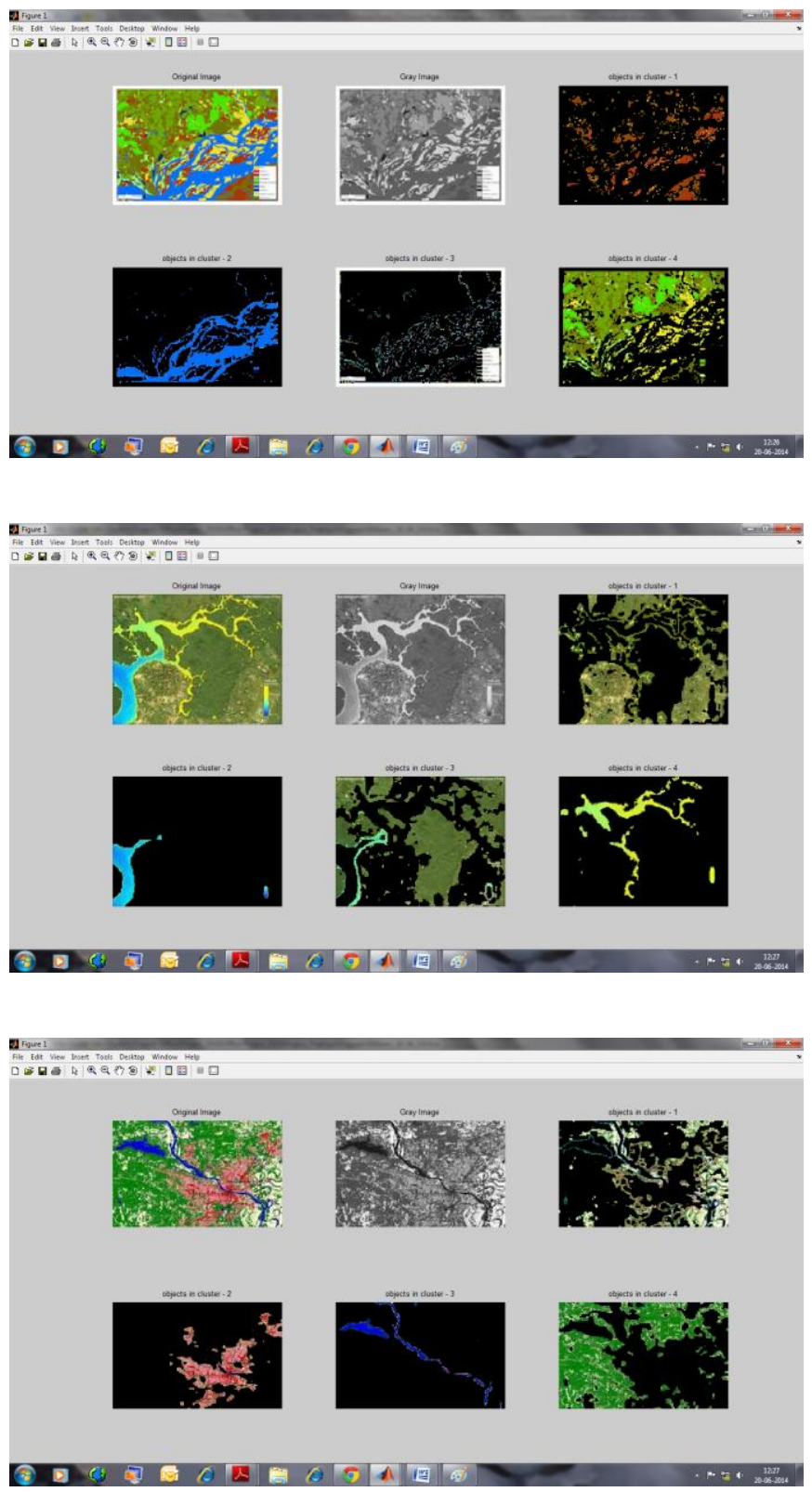

Figure 4.1 Results of Different Landscapes before \& After Applying the BBO Algorithm

\section{FUTURE SCOPE}

The presented algorithm has been tuned primarily for satellite images. However, same can be used for other landscape images as well. The speed of operation goes down as the size of the image increases. There is scope for working in enhancing the speed of operation of the algorithm as the size of image increases. Also the work is implemented in matlab version 7.5. The same algorithm may be implemented in any hardware oriented language for real time image processing applications.

\section{REFERENCES}

[1] Carlos Rueda," Modeling Satellite Image Streams for Change Analysis", Proceedings of the 15th International Symposium on Advances in Geographic Information Systems ACM GIS 2007 ACMGIS'07 November 7-9, 2007, Seattle, WA ACM 978-1-59593-914-2/07/11

[2] Dr.P.Subashini," Quantitative performance evaluation on segmentation methods for SAR ship images", Compute'10, Jan 22-23, 2010, Bangalore, Karnataka, India ACM 978-1-4503-0001-8/00/0010

[3] Prof. Dr. P. K. Srimani," Analysis and Evaluation of Classifiers Using Multi-Temporal Images in Land Use and Land Cover Mapping", A2CWiC 2010, September 16-17, 2010, India 978-1-4503-0194-7/10/0009

[4] Dr.P.Subashini," A combined preprocessing scheme for texture based Ice classification from SAR images", A2CWiC 2010, September 16-17, 2010, India 978-14503-0194-7/10/0009

[5] Kanchan Bahirat,'Matte Based Generation of Land Cover Maps", ICVGIP '12, December 16-19, 2012, Mumbai, India ACM 978-1-4503-1660-6/12/12

[6] Abida Najab," Principal Component Analysis Based Classification of Settlements in Satellite Images", FIT'09, December 16-18, 2009, CIIT, Abbottabad, Pakistan. ACM 978-1-60558-642-7/09/12

[7] Lavika Goel," Performance Governing Factors of Biogeography Based Land Cover Feature Extraction: An Analytical Study", 2011 World Congress on Information and Communication Technologies 978-1-4673-0125-1@ 2011 IEEE (pp 165-170)

[8] Triloki Pant," Application of Fractal Parameters for Unsupervised Classification of SAR images: A Simulation based Study", IITM'10, December 28-30, 2010, Allahabad, UP, India. ACM 978-1-4503-04085/10/12 (pp 45-50)

[9] V Turkar," Polarimetric SAR Image Classification by Using Artificial Neural Network", International Conference and Workshop on Emerging Trends in Technology (ICWET 2010) - TCET, Mumbai, India ICWET'10, February 26 - 27, 2010, Mumbai, Maharashtra, India. ACM 978-1-60558-812-4 (pp 48-52)

[10] Bruno M. Carvalho," Texture Fuzzy Segmentation using Adaptive Affinity Functions", SAC'12 March 25-29, 2012, Riva del Garda, Italy. ACM 978-1-4503-0857$1 / 12 / 03$ (pp 51-53)

[11] Ruochen Liu," A Multiobjective Immune Clustering Ensemble Technique Applied to Unsupervised SAR Image Segmentation", CIVR '10, July 5-7, Xi'an China ACM 978-1-4503-0117-6/10/07 (pp 158-165) 
[12] Jiang Li," Remote Sensing Image Information Mining with HPC Cluster and DryadLINQ", 49th ACM Southeast Conference, March, 24-26, 2011, Kennesaw, GA, USA. ACM 978-1-4503-0686-7/11/03 (pp 227-232)

[13] H.B. Kekre," SAR Image Segmentation using cooccurrence matrix and slope magnitude", International Conference on Advances in Computing, Communication and Control (ICAC3'09) ICAC3'09, January 23-24, 2009, Mumbai, Maharashtra, India. Copyright 2009 ACM 978-1-60558-351-8 (pp 368-372)
[14] Xiuli Ma," Spectral Clustering Ensemble for Image Segmentation", GEC'09, June 12-14, 2009, Shanghai, China. ACM 978-1-60558-326-6/09/06 (pp 415-420)

[15] Jiang Li," Hierarchical Land Cover Information Retrieval in Object-Oriented Remote Sensing Image Databases with Native Queries", ACMSE 2007, March 23-24, 2007, Winston-Salem, North Carolina, USA ACM 978-1-59593-629-5/07/0003 (pp 467-472)

[16] Mark Hauschild," Image Segmentation using a Genetic Algorithm and Hierarchical Local Search", GECCO'12, July 7-11, 2012, Philadelphia, Pennsylvania, USA ACM 978-1-4503-1177-9/12/07 (pp 633-639) 